\title{
ANALYTICAL MODEL FOR FRACTURE BEHAVIORS OF PSEUDO STRAIN-HARDENING CEMENTITIOUS COMPOSITES
}

\author{
Petr KABELE ${ }^{1}$ and Hideyuki HORII ${ }^{2}$ \\ ${ }^{1}$ Member of JSCE, Ph.D., Research Associate, Dept. of Civil Engineering, The University of Tokyo, (Hongo 7-3-1, \\ Bunkyo-ku, Tokyo 113, Japan) \\ ${ }^{2}$ Member of JSCE, Ph.D., Associate Professor, Department of Civil Engineering, The University of Tokyo
}

\begin{abstract}
In the present paper we propose a simple analytical model for fracture analysis of short fiber reinforced cementitious composites designed to undergo distributed multiple cracking prior to formation of a localized crack under tensile and shear loads. The composite in multiple cracking state is idealized as a homogenous and continuous material, with cracks being represented by cracking strain. A discrete crack model is used for localized cracks. The model is implemented into FEM code and experimental results are reproduced proving validity of the proposed model.
\end{abstract}

Key Words: cementitious composites, short fibers, multiple cracking, localized cracking, analytical model, plasticity, discrete crack model

\section{INTRODUCTION}

In recent years, a big attention has been paid to development of high performance structural materials for civil engineering applications. A group of such materials, called engineered cementitious composites, has been recently developed by $\mathrm{Li}$ and co-workers 1), 2), 3). Engineered cementitious composites (ECCs) are cement based materials reinforced with short, randomly oriented and randomly distributed fibers. In contrast to other, usually quasi-brittle, short fiber cementitious composites, the structure of ECCs is designed in such a way, that under tensile and shear loads they undergo multiple cracking accompanied by overall pseudo strain-hardening behavior prior to formation of a localized crack. Consequently, the fracture process zone in ECCs is not concentrated in a single plane or narrow band around the localized crack, but spreads over large volume of the material. As a result of the extensive cracking off the main crack plane, ECCs exhibit high fracture energy and critical tensile strain up to two orders of magnitude higher than usual cementitious composites with short fibers.

In the present paper, we propose an analytical model for the fracture analysis of structures made with use of ECCs. The motivation for developing such an analytical model for ECCs can be summarized as follows:
- Feedback to experimental studies: results of numerical analyses can be used to estimate the optimal setup for actual experiments, to verify experimental results, or can reveal existence of phenomena which should be further experimentally investigated.

- Numerical experiments: physical experiments which are too expensive or difficult to carry out can be replaced by numerical experiments (this concerns, for example, investigation of size effect which involves large specimens).

- Structural analysis: the analytical model can be used to conduct pilot studies on applicability and feasibility of ECCs in structural members.

In order to be able to accomplish these tasks, the model must adequately represent the presence of both multiple and localized cracking in ECCs. Furthermore, the model must be suitable for implementation into an FEM code so that structures of general geometries can be analyzed.

The earlier theoretical studies related to ECCs, e.g., by $\mathrm{Li}^{4)}$ and $\mathrm{Li}$ and Leung ${ }^{5)}$, mostly focus on the mechanics of a single crack with fiber bridging. These studies clarify the conditions for existence of multiple cracking. They also provide link between the composite micromechanical parameters (such as, fiber aspect ratio, fiber and matrix elastic moduli, fracture toughness of matrix, fiber-matrix interfacial bond strength, etc.) and the bridging stress to crack 


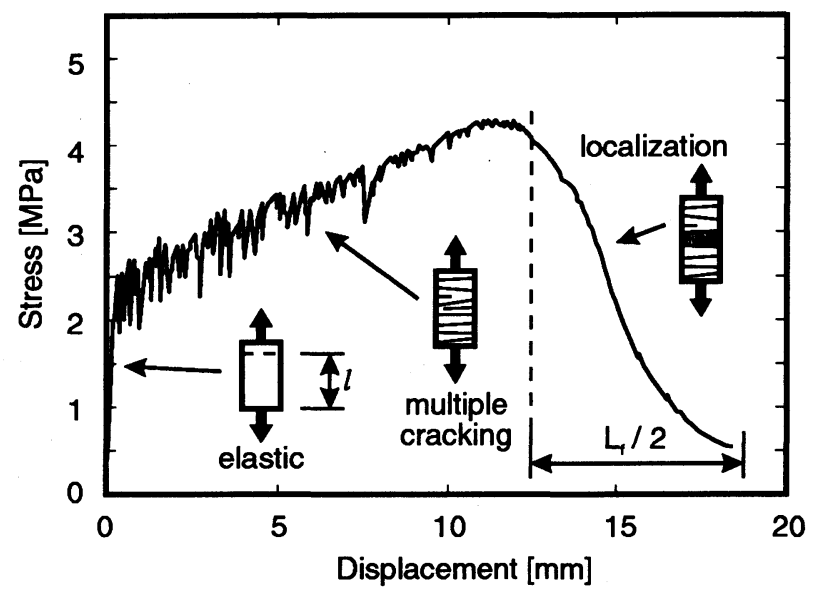

Fig. 1 Experimental ${ }^{8)}$ uniaxial stress-displacement curve for ECC with $2 \%$ by volume of polyethylene fibers

opening displacement (COD) relationship. Nevertheless, none of these studies provides an analytical model that could be directly implemented into an FEM code. Other well-established fracture models for cementitious materials, such as, the fictitious crack model (FCM) proposed by Hillerborg et al. ${ }^{6}$, do not consider the presence of the off-maincrack-plane multiple cracking zone and by themselves alone can not be used for analysis of ECCs.

In the present study, we idealize the composite undergoing multiple cracking as a homogenous and continuous material, with cracks being represented by additional (cracking) strain. With help of the incremental theory of plasticity we derive an incremental constitutive law for the composite in multiple cracking state, which is then directly used in an FEM program.

The localized cracks with bridging are modeled as discrete discontinuities in the displacement field. The discrete crack model is implemented into the FEM program with help of a cracked element developed by Nanakorn and Horii ${ }^{\text {? }}$.

\section{FRACTURE BEHAVIOR OF ECCS UNDER TENSILE AND SHEAR LOADS}

The mechanism of tensile cracking in ECCs can be clarified when analyzing the results of uniaxial tension tests conducted by $\mathrm{Li}^{1)}$ and Maalej et al. ${ }^{8)}$. Fig. 1 shows a typical uniaxial stress-displacement curve for an ECC specimen. The ECC consisted of cement paste reinforced with $2 \%$ by volume of Spectra polyethylene fibers. The fiber length and diameter were $12.7 \mathrm{~mm}$ and $38 \mu \mathrm{m}$, respectively. The experiments revealed that after formation of the first crack at the load magnitude equal to the first crack strength of the composite $\sigma_{f c}$, the material continued to sustain increasing loading. The increase in load was accompanied by formation of additional subparallel cracks - a process called multiple cracking. Photographs taken during the experiment show that the cracks were more or less normal to the direction of the applied load. The pictures also suggest that during the multiple cracking, both crack openings and crack density were increasing with increasing load. As soon as the load reached the value hereafter referred to as maximum bridging stress $\sigma_{m b}$, the specimen started to exhibit overall softening behavior. The transition into softening was associated with localization of the fracture into a single crack - apparently the one that had had previously the largest COD. In the other words, after the peak, only one of the existing cracks continued to open while the other ones were undergoing unloading. The magnitude of the load during the post-peak part of the uniaxial test was controlled by the bridging stress transmitted across the localized crack. This stress decreased gradually with increasing COD of the localized crack, following a tension softening relationship.

$\mathrm{Li}^{4)}$ has shown that assuming random fiber distribution, the bridging stress should diminish when the value of COD reaches one half of the fiber length. It is seen in Fig. 1 that if we subtract this value (in this case $L_{f} / 2=6.35 \mathrm{~mm}$ ) from the total displacement of the specimen when the stress just becomes equal to zero, we get approximately the displacement at the peak load. This means, that the portion of the total displacement associated with the multiple cracking is not recoverable upon unloading the specimen to zero stress. 
It has to be noted that even though the total deformation of the specimen due to the multiple cracking is relatively large, the high density of multiple cracks (several cracks per centimeter) ensures that their widths are very small, usually around $0.1 \mathrm{~mm}$.

As $\mathrm{Li}$ and Leung ${ }^{5)}$ explain, multiple cracking is possible due to the properly designed fiber reinforcement, which ensures that after a crack is formed, the bridging fibers undergo frictional debonding while transmitting increasing stress across the crack plane. The transition to softening mode is attributed to completed debonding of the bridging fibers and their slippage from the matrix.

$\mathrm{Li}$ et al. ${ }^{9)}$ also experimentally examined the structural response of shear beams made of ECCs. The specimens were reinforced by steel bars along the top and bottom surfaces in order to avoid flexural failure, but no conventional shear reinforcement was used. The ECC beams exhibited high ductility due to diagonal multiple cracking which occurred over a large volume of the material. The experiments confirmed that the behavior of ECCs under biaxial tension-compression stress state is governed by the same phenomena that control the uniaxial behavior; that is, multiple cracking in direction normal to the maximum principal stress and consequent localization into the crack with the largest COD. The experimental results also hinted that the compressive stress acting parallel to the cracks might positively influence the material strength. However, the experimental evidence so far is not sufficient to establish any relationship between the stress parallel to the direction of the cracks and the first crack strength, stress transfer capability of the bridging fibers or the ultimate strength of the bridging. For this reason we will neglect this effect throughout the present study.

\section{ANALYTICAL MODEL FOR ECCS}

Due to the randomness of fiber orientation and distribution and short fiber lengths, ECCs are macroscopically homogenous and isotropic. The mechanical behavior of ECCs is dominated by the cracking they undergo in the process of loading. Therefore we treat ECCs in the proposed model as homogenous materials with cracks. Due to the different nature of the two types of cracking that occur in ECCs, we use different modeling approaches for distributed multiple cracking and for localized cracking.

\section{(1) Multiple cracking}

Multiple cracking is characterized by formation of a large number of relatively uniformly distributed subparallel cracks. The crack density is high while the crack widths are relatively small. In such a situation, it would not be practical to treat each crack separately. Instead, we idealize the composite undergoing multiple cracking as a continuous material with additional strain, called cracking strain, which represents the crack openings and density.

As we have discussed in the previous section, the deformation due to the multiple cracking is not recoverable upon unloading, which means that the cracking strain is inelastic. Considering further that the material in the multiple cracking state exhibits pseudo strain-hardening behavior, strain-hardening theory of plasticity appears to be a suitable tool for modeling of multiple cracking in ECCs. The task is then to find a proper yield function and hardening rule.

\section{a) Yield function and hardening rule}

It has been discussed earlier that multiple cracking is initiated on planes normal to the maximum principal stress when its magnitude reaches the first crack strength. Keeping in mind that when using the associated flow rule, the yield function not only determines the conditions for initiation of plastic yielding (or in our case multiple cracking) but also the direction of plastic (or cracking) strain increment, we define the yield surface in 2-D stress state by the Rankine yield function:

$$
\begin{gathered}
F \equiv \frac{\sigma_{x x}^{*}+\sigma_{y y}^{*}}{2}+ \\
\sqrt{\left(\frac{\sigma_{x x}^{*}-\sigma_{y y}^{*}}{2}\right)^{2}+\left(\frac{\sigma_{x y}^{*}+\sigma_{y x}^{*}}{2}\right)^{2}}-\sigma_{f c}=0
\end{gathered}
$$

where $\sigma_{f c}$ is the first crack strength and $\sigma_{x x}^{*}, \sigma_{y y}^{*}, \sigma_{x y}^{*}$ and $\sigma_{y x}^{*}$ are defined by the kinematic hardening rule as follows:

$$
\sigma_{i}^{*}=\sigma_{i}-\alpha_{i}
$$

Hereafter $i$ equals successively to $x x, y y, x y$ and $y x$. Then $\sigma_{i}$ is a vector consisting of in-plane components of the stress tensor. The vector $\alpha_{i}$ is defined by:

$$
d \alpha_{i}=h d \varepsilon_{i}^{c}
$$


in which $d \varepsilon_{i}^{c}$ is the cracking strain increment vector and $h$ is a function of the total cracking strain which reflects the material hardening behavior.

It is seen in Eq. (2) that before any multiple cracking occurs (i.e., $\alpha_{i}=0$ ), $\sigma_{i}^{*}$ is equal to the vector of in-plane components of the stress tensor. Therefore the first two terms of the yield function (Eq. (1)) initially correspond to the magnitude of the maximum principal stress in the $x-y$ plane. Eq. (1) thus satisfies the condition that the multiple cracking is initiated when the maximum principal stress is equal to the first crack strength. It should be noted that we use Eq. (1) for both plane stress and plane strain; in the latter case we assume that the out-ofplane stress component $\sigma_{z z}$ induces no cracking on planes parallel to $x-y$.

The associated flow rule, which we employ in the present model, implies that the cracking strain increment is proportional to the normal vector of the yield surface in the stress space:

$$
d \varepsilon_{i}^{c}=d \lambda \frac{\partial F}{\partial \sigma_{i}}
$$

where $d \lambda$ is a non-negative scalar factor of proportionality. The normal vector $\partial F / \partial \sigma_{i}$ can be evaluated when substituting Eq. (1) as follows:

$$
\begin{aligned}
& \frac{\partial F}{\partial \sigma_{x x}}=\frac{1}{2}+\frac{\sigma_{x x}^{*}-\sigma_{y y}^{*}}{4 \sqrt{\left(\frac{\sigma_{x x}^{*}-\sigma_{y y}^{*}}{2}\right)^{2}+\left(\frac{\sigma_{x y}^{*}+\sigma_{y x}^{*}}{2}\right)^{2}}} \\
& \frac{\partial F}{\partial \sigma_{y y}}=\frac{1}{2}-\frac{\sigma_{x x}^{*}-\sigma_{y y}^{*}}{4 \sqrt{\left(\frac{\sigma_{x x}^{*}-\sigma_{y y}^{*}}{2}\right)^{2}+\left(\frac{\sigma_{x y}^{*}+\sigma_{y x}^{*}}{2}\right)^{2}}} \\
& \frac{\partial F}{\partial \sigma_{x y}}=\frac{\partial F}{\partial \sigma_{y x}}=\frac{\sigma_{x y}^{*}+\sigma_{y x}^{*}}{\sqrt[4]{\left(\frac{\sigma_{x x}^{*}-\sigma_{y y}^{*}}{2}\right)^{2}+\left(\frac{\sigma_{x y}^{*}+\sigma_{y x}^{*}}{2}\right)^{2}}}
\end{aligned}
$$

It is easy to show that if Eq. (4) and Eqs. (5) hold, the maximum and minimum principal values of cracking strain increment are:

$$
\begin{aligned}
& \left(d \varepsilon^{c}\right)_{1}=d \lambda \\
& \left(d \varepsilon^{c}\right)_{2}=0
\end{aligned}
$$

respectively, with the angle of the principal cracking strain increment $\theta^{d \varepsilon}$ being defined by:

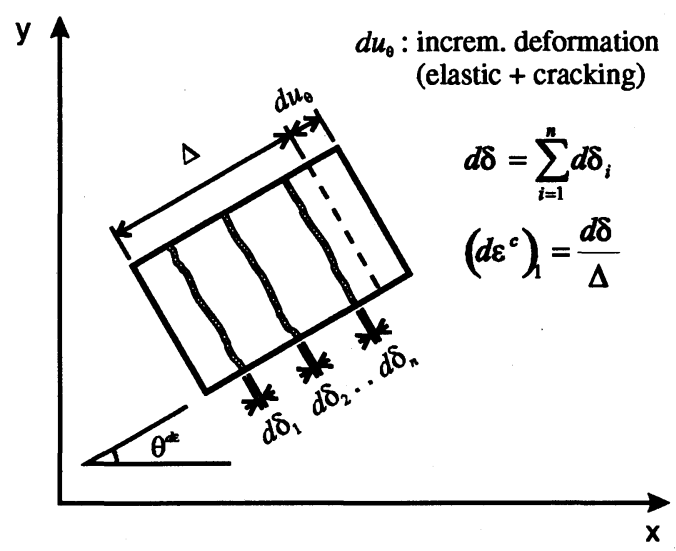

Fig. 2 Cracking strain increment to COD increment relationship

$$
\tan \left(2 \theta^{d \varepsilon}\right)=\frac{\sigma_{x y}^{*}+\sigma_{y x}^{*}}{\sigma_{x x}^{*}-\sigma_{y y}^{*}}
$$

Fig. 2 shows that the incremental cracking strain $\left(d \varepsilon^{c}\right)_{1}$ in fact represents incremental normal crack opening displacements of a set of cracks oriented at the right angle to the direction $\theta^{d \varepsilon}$. Note that, consistently with this interpretation, the zero value of $\left(d \varepsilon^{c}\right)_{2}$ ensures that the cracks do not contribute to the deformation in direction normal to $\theta^{d \varepsilon}$.

As angle $\theta^{d \varepsilon}$ may change throughout the loading, an infinite number of sets of multiple cracks, each oriented at a different angle, may occur at any point of the material. It is understood that this is a certain simplification of the real material behavior. In the reality, a small change of the principal stress direction would cause sliding of the existing cracks. But if the change in the principal stress direction exceeds a certain limit, formation of a new set of cracks would occur. However, to implement these mechanisms into the analytical model would require more experimental knowledge on the sliding behavior of cracks than is currently available.

It has been discussed earlier that before multiple cracking has occurred, $\sigma_{i}^{*}$ is equal to $\sigma_{i}$. Thus angle $\theta^{d e}$ is initially equal to the angle of maximum principal stress $\theta^{\sigma}$ which is defined by the following equation:

$$
\tan \left(2 \theta^{\sigma}\right)=\frac{\sigma_{x y}+\sigma_{y x}}{\sigma_{x x}-\sigma_{y y}}
$$




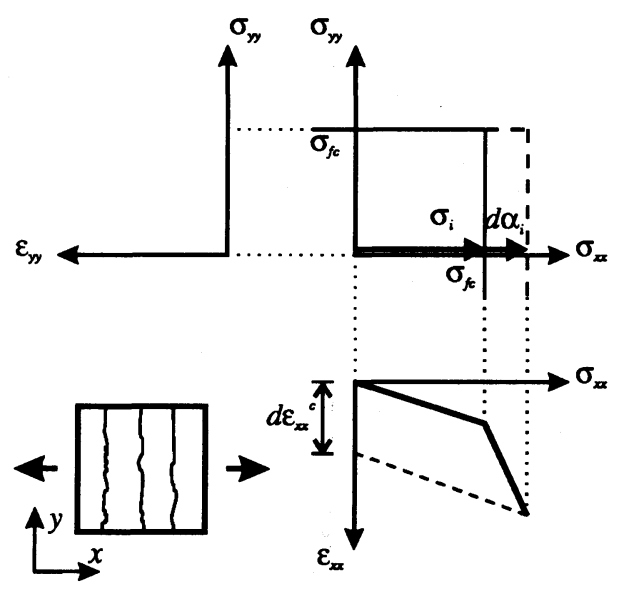

(a)

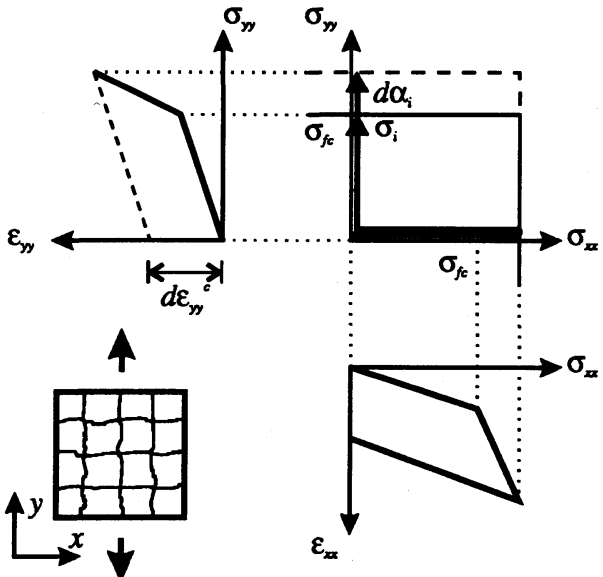

(b)

Fig. 3 Example of two-directional loading; (a) loading in $x$-direction; (b) unloading and reloading in $y$-direction

The proposed model therefore correctly represents the fact that the first set of multiple cracks is initiated on planes which are normal to the maximum principal stress.

The kinematic hardening rule defined by Eq. (2) implies that when the hardening takes place, the yield surface translates in the stress space in the direction of vector $d \alpha_{i}$ or, considering Eq. (3), in the direction of the cracking strain increment $d \varepsilon_{i}^{c}$. If the isotropic hardening rule was used, the yield surface would instead expand uniformly in the stress space. To explain why we employ the kinematic hardening rule as opposed to the isotropic hardening rule, let us consider an example of two-directional loading as shown in Fig. 3. The figure shows the loading path and the yield surface in the $\sigma_{x x}-\sigma_{y y}$ plane (top right), the stress-strain curves for $x$ and $y$ directions (bottom right and top left, respectively) and schematic sketch of the cracked specimen (bottom left), at two different stages of loading (a) and (b). Let us assume that the material is first uniaxially loaded in the direction of $x$-axis - see Fig. 3 (a). As the load reaches the first crack strength $\sigma_{f c}$, multiple cracking starts on planes normal to the $x$-axis resulting in incremental cracking strain whose only non-zero component is $d \varepsilon_{x x}^{c}$, as required by the associated flow rule and the shape of the yield surface. This is reflected in the stress space by translating the original yield surface (which appears as two lines starting from point $\left[\sigma_{f c}, \sigma_{f c}\right]$ and running parallel to $\sigma_{x x}$ - and $\sigma_{y y}$-axes) by incremental vector $d \alpha_{i}$, which is parallel to the $\sigma_{x x}$-axis. It is seen in Fig. 3 (b) that if the material is unloaded to zero stress and reloaded in the perpendicular direction (i.e., along the $y$-axis), the yield surface is reached when $\sigma_{y y}=\sigma_{f c}$. Further loading results in cracking strain increment parallel to the $\sigma_{y y}$ axis, i.e., formation of a new set of cracks that are perpendicular to the existing ones.

If the isotropic hardening rule was used, the initial loading in the $x$-direction would cause uniform expansion of the yield surface. Then, upon reloading in the $y$-direction, no multiple cracking would take place until the yield surface is reached at a load level equal to the maximum load attained previously in the $x$-direction, which is higher than $\sigma_{f c}$.

As a result we can conclude that the present model with kinematic hardening rule reflects the assumption that for a given direction, neither the first crack strength, nor the hardening response of the cracked material, are affected by the stress or cracking strain perpendicular to this direction.

Once the yield function and hardening rule are set, the elasto-plastic incremental stress-strain relationship can be derived using standard procedures of incremental theory of plasticity as shown, for example, by Chen and Han ${ }^{10}$. It is noted that the elasto-plastic constitutive law is used only when the material undergoes plastic loading, i.e., the following condition is satisfied:

$$
\frac{\partial F}{\partial \sigma_{i}} d \sigma_{i}>0
$$




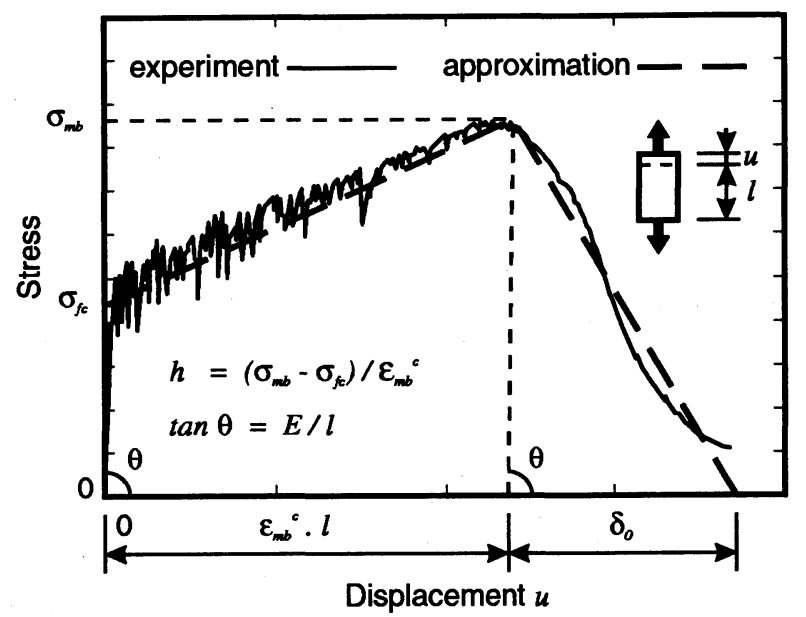

Fig. 4 Determination of material parameters from approximated uniaxial stress-displacement curve

where we apply summation rule over index $i=x x$, $y y, x y$ and $y x$. If inequality (9) is not satisfied, elastic stress-strain relation is used.

\section{b) Treatment of the yield surface singularity}

The only case which requires a special attention while deriving the incremental elasto-plastic stressstrain relation is when:

$$
\sigma_{x x}^{*}=\sigma_{y y}^{*}=\sigma_{f c} \text { and } \sigma_{x y}^{*}=\sigma_{y x}^{*}=0
$$

The equalities in Eq. (10) define the singular point of the yield surface. It is obvious that if we substitute Eq. (10) into Eqs. (5) we obtain indefinite expressions $0 / 0$, which means that the normal vector to the yield surface can not be uniquely determined.

Various methods for treatment of the yield surface singularities have been proposed in the literature ${ }^{10), 11)}$. If the singularity is formed by intersection of several smooth surfaces, then the plastic strain vector can be determined as linear combination of normal vectors of the adjacent surfaces. This approach, however, involves some difficulties in case of the Rankine yield function ${ }^{11}$. Another way is to replace the original yield function in a certain range around the singular point by another, smooth, function. Nevertheless, this approach requires introduction of an additional parameter (the size of the range to be replaced) and of the smoothing function, which are related rather to the mathematical modeling then to the material mechanical behavior.

In order to overcome these shortcomings we adopt yet another way, which is described hereafter. The consistency condition requires that:

$$
d F=\frac{\partial F}{\partial \sigma_{i}} d \sigma_{i}+\frac{\partial F}{\partial \alpha_{i}} d \alpha_{i}=0
$$

It is obvious from Eq. (2) that:

$$
\frac{\partial F}{\partial \alpha_{i}}=-\frac{\partial F}{\partial \sigma_{i}}
$$

Then substituting Eq. (3) and Eq. (12) into Eq.(11) we get:

$$
d F=\frac{\partial F}{\partial \sigma_{i}}\left(d \sigma_{i}-h d \varepsilon_{i}^{c}\right)=0
$$

It can be shown that if the singular point defined by Eq. (10) is approached along the yield surface $F=0$ from any given direction, then the normal vector $\partial F / \partial \sigma_{i}$ can be determined uniquely depending on this direction. In order to satisfy Eq. (13) in the singular point for any $\partial F / \partial \sigma_{i}$, i.e., if we approach the singular point from any direction, we must put:

$$
\left(d \sigma_{i}-h d \varepsilon_{i}^{c}\right)=0
$$

Then the incremental stress-strain relationship can be obtained using Eq. (14), decomposition of the strain increment into elastic and plastic (cracking) part and elastic relationship between the stress increment and elastic strain increment as follows:

$$
d \sigma_{i}=\left(\frac{1}{h} \delta_{i j}+D_{i j}^{e}\right)^{-1} d \varepsilon_{j}
$$


where $\delta_{i j}$ is the Kronecker delta operator and $D_{i j}^{e}$ is the elastic compliance matrix.

It should be noted that the Drucker's stability postulate implies that at the corner of the yield surface, the plastic strain vector must lie between the adjacent normals, which is also the condition for applicability of Eq. (15).

\section{c) Material parameters}

Eq. (1) and Eq. (3) suggest that the model of multiple cracking is characterized by two material parameters - first crack strength $\sigma_{f c}$ and function $h$ which reflects the shape of the hardening part of the stress-strain curve.

As shown in Fig. 4, both material parameters $\sigma_{f c}$ and $h$ can be easily determined from a uniaxial stress-strain curve. The hardening part of the stressstrain curve can be fairly well approximated by a linear relationship, which allows us to use constant $h$.

\section{(2) Localized crack with bridging}

In the model for multiple cracking explained in the previous subsection, the cracking strain represents the density and widths of the multiple cracks. The direction of the maximum principal cracking strain then can be interpreted as the direction normal to the most developed set of multiple cracks. Because the experimental results suggest that the focus of localization is in the one of the multiple cracks that has the largest opening displacement, we employ the following condition for formation of the localized crack. The localized crack is initiated on the plane normal to the maximum principal cracking strain when its magnitude reaches a certain critical value $\varepsilon_{m b}^{c}$. It has to be noted that the above condition is not sufficient to reproduce the fracture localization in the case of a perfectly homogenous body with a uniform cracking strain field. In such a situation, the localization would have to decided by employing the thermodynamics based theory for localization phenomena proposed by Horii and Okui ${ }^{12)}$.

The localized cracks are characterized by large opening displacements and by existence of bridging. Therefore, the cracks are modeled as discrete discontinuities in the displacement field with the effect of bridging being represented by traction applied to the crack surfaces.

Generally, both normal and tangent components of this traction are related to the relative displacements of the crack surfaces. However, for the lack of experimental data on the shear behavior of the localized cracks, we assume that once the localized crack is formed, the normal bridging traction is related only to the normal COD, while the shear traction stays constant. It is noted that the latter assumption is adopted only for simplicity and has to be revised if cracks undergoing relative shear displacement are to be correctly represented. The normal traction decreases with increasing normal COD according to the tension softening relationship, which is given in incremental form as follows:

$$
d t_{n}=s d \delta_{n}
$$

where $d t_{n}$ stands for increment of normal traction; $s$ is the slope of the tension softening curve; and $d \delta_{n}$ is incremental normal COD.

The expression in Eq. (16) is valid only for cracks that are opening. There is no experimental information available about the response of the localized cracks in ECCs during the crack closure. Thus we use the widely accepted assumption that once the crack starts to close, the normal traction is linearly decreasing with decreasing normal COD so that it reaches zero at zero COD (neglecting the crack opening displacement gained during the multiple cracking).

It is noted that, on the contrary to the model for multiple cracking, once the localized crack is formed at certain location, its direction is fixed and no other localized crack opens at the same location. It is possible however, that the material undergoes multiple cracking in the direction perpendicular to the localized crack.

The model for localized cracks is defined by two parameters: critical cracking strain $\varepsilon_{m b}^{c}$ and the tension softening curve. The value of $\varepsilon_{m b}^{c}$ is determined from the result of the uniaxial tension test as shown in Fig. 4. Fig. 4 suggests, that the postpeak part of the stress-displacement curve is almost linear, which allows us to set $s$ constant. The value of $s$ is then calculated as negative ratio between the normal traction transmitted across the crack when it is initiated at the critical cracking strain $\varepsilon_{m b}^{c}$ and the critical crack width $\delta_{o}$, which is determined from the uniaxial stress-displacement curve as shown in Fig. 4.

\section{NUMERICAL RESULTS}

The analytical model described in Section 3. has been implemented into an FEM program. The model for multiple cracking has been implemented by means of incremental constitutive law. In the case of the localized crack model, we employ the cracked 
Table 1 Material parameters for composites with various fiber volume fractions

\begin{tabular}{|c|c|c|c|c|c|c|}
\hline$V_{f}[\%]$ & $E[\mathrm{GPa}]$ & $v[-]$ & $\sigma_{f c}[\mathrm{MPa}]$ & $\varepsilon_{m b}^{c}[\%]$ & $\sigma_{m b}[\mathrm{MPa}]$ & $\delta_{0}[\mathrm{~mm}]$ \\
\hline 0.8 & 10 & 0.2 & 2.0 & 2.27 & 2.85 & 6.27 \\
\hline 1.0 & 10 & 0.2 & 2.0 & 2.82 & 3.05 & 6.17 \\
\hline 2.0 & 22 & 0.2 & 2.2 & 5.78 & 4.32 & 6.62 \\
\hline 3.0 & 10 & 0.2 & 2.5 & 6.15 & 4.97 & 5.28 \\
\hline
\end{tabular}

element developed by Nanakorn and Horii "). As all the governing equations are in incremental form, Euler method based algorithm is used to integrate the incremental solutions.

In order to verify the performance of the proposed model we have used it to reproduce some of experiments conducted on ECCs by $\mathrm{Li}$ and Hashida ${ }^{3)}$ and Maalej et al. ${ }^{8)}$. These studies provide experimental results for two types of test specimens: uniaxial tension (UT) specimens and double cantilever beam (DCB) specimens of various sizes. The material used in these experiments is a cement mortar reinforced with polyethylene fibers. The fiber volume fraction $V_{f}$ is fixed equal to $2 \%$ in ref. 3 ) and varying between $0.2 \%$ and $4 \%$ in ref. 8 ).

\section{(1) Overall response and cracking behavior}

At first, we determined the material parameters from the experimental uniaxial stress-displacement curve of composite with $V_{f}$ equal to $2 \%$ (Fig. 1). The material parameters are listed in Table 1. Using these parameters we analyzed the medium size DCB specimen described in ref. 3). The specimen geometry is shown in Fig. 6; the specimen dimensions are listed in Table 2. The notch length used in this analysis was $a_{1}=11.7 \mathrm{~cm}$.

The specimen was discretized by finite element mesh, which initially consisted of 2530 isoparametric quadrilateral 4-node elements. Some of the elements were during the computation automatically changed into cracked elements as a result of the localized crack propagation. The mesh is shown in Fig. 5. It is noted that due to the material hardening behavior in the multiple cracking state and due to the bridging stress transfer along the localized crack, the stress at the crack tip is bounded and stress singularity vanishes. Thus, as far as the overall behavior is considered, the effect of the stress concentration at the crack tip is minor compared to the effect of gradually expanding zone of multiple cracking and the effect of fiber bridging across the localized crack. Accordingly, we employ almost uniform mesh to reduce the influence of meshing on the development of the multiple cracking zone. Also, sufficient number of elements in the ligament is necessary to
Table 2 Dimensions of the DCB specimens

\begin{tabular}{|c|c|c|c|c|}
\hline size & $\mathrm{h}[\mathrm{cm}]$ & $\mathrm{w}[\mathrm{cm}]$ & $\mathrm{a}_{1}[\mathrm{~cm}]$ & $\mathrm{a}_{2}[\mathrm{~cm}]$ \\
\hline small & 15.3 & 12.7 & 6.5 & 7.4 \\
\hline medium & 30.0 & 31.0 & 11.7 & 14.7 \\
\hline large & 58.5 & 49.0 & 13.4 & 21.5 \\
\hline
\end{tabular}

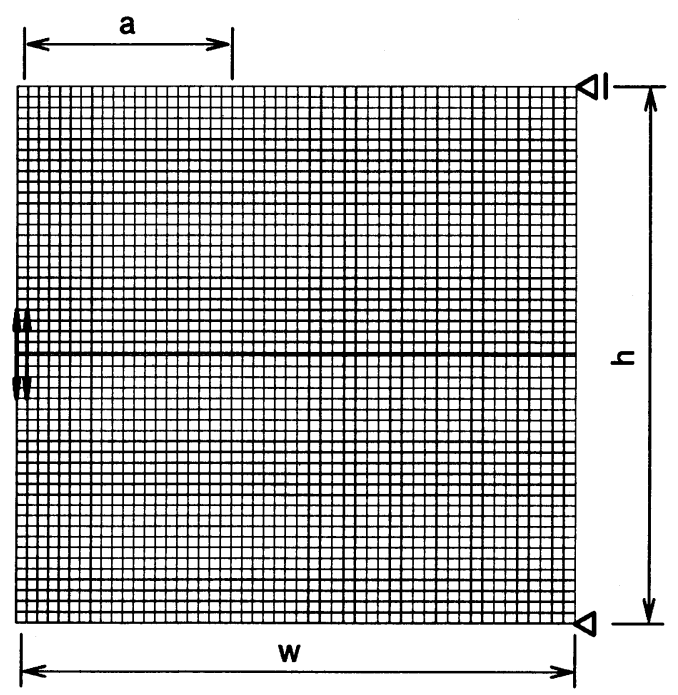

Fig. 5 Finite element discretization of the medium size DCB specimen

assure proper approximation of the COD along the localized crack once it propagates.

Fig. 6 compares the experimental ${ }^{3)}$ and analytical load-displacement curves. The figure suggests that the proposed model could reproduce the presence of the significant pre-peak nonlinearity, the displacement at the peak and the post-peak branch of the load-displacement curve. The model, however, predicted higher load at the peak. Possible reasons for this discrepancy will be discussed later.

Fig. 7 shows the distribution of the cracking strain and evolution of the localized crack at different loading stages indicated by points A and B in Fig. 6. Fig. 7 (a) shows that before the bend-over point of the load-displacement curve, cracking is concentrated near the original notch tip. However, upon reaching the hardening portion of the curve, the multiple 


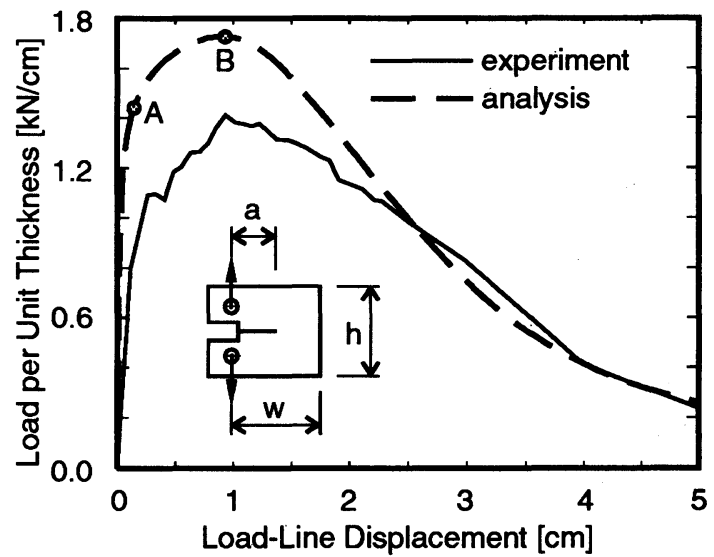

Fig. 6 Load-displacement curves for medium size DCB specimen $\left(V_{f}=2 \%\right)$

cracking spreads rapidly around the notch tip while the evolution of the localized crack is relatively slow. It is seen in Fig. 7 (b) that at the peak load, the zone of multiple cracking has an onion like shape and extends almost to the specimen boundaries, which is consistent with the experimental result. As the direction of maximum principal cracking strain can be interpreted as normal to the most developed multiple cracks, Fig. 7 (c) shows that the multiple cracking pattern as well as the extension of the localized crack are also in good agreement with the photographs of the cracked specimen provided in ref. $3)$.

\section{(2) Effect of fiber volume fraction}

Maalej et al. ${ }^{8)}$ studied the influence of the fiber volume fraction on the fracture energy of ECCs. The authors observed that the total fracture energy $J_{c}$ of ECCs is composed of two components. The first, called bridging fracture energy $J_{b}$, is associated with the fiber pull-out process at the main fracture plane. The second one, denoted as $J_{m}$, comes from the distributed multiple cracking. The authors of ref. 8) proposed a J-integral based technique to evaluate the total fracture energy $J_{c}$ and the component $J_{b}$. The total fracture energy is calculated using loaddisplacement curves of two DCB specimens which differ only in the original notch length. The bridging fracture energy is obtained from the post-peak portion of a uniaxial load-displacement curve. The fracture energy contributed by the multiple cracking $J_{m}$ is then obtained as a difference between $J_{c}$ and $J_{b}$.

We tried to reproduce the effect of fiber volume fraction on the composite fracture energy with the proposed model. The fiber volume fractions that we selected for our analyses were $0.8 \%, 1 \%, 2 \%$ and (a)

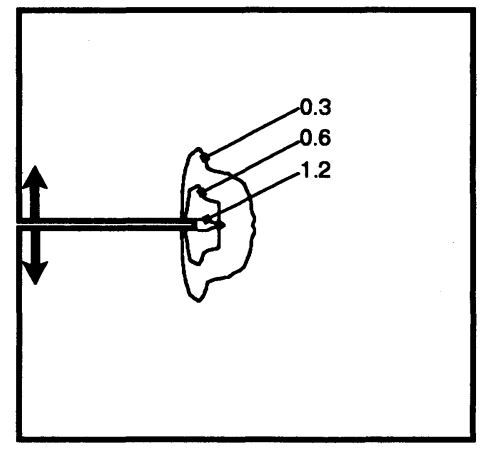

(b)

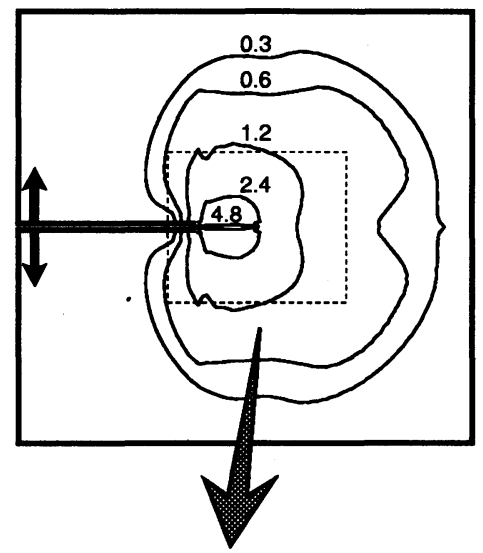

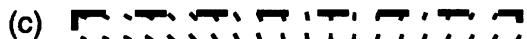

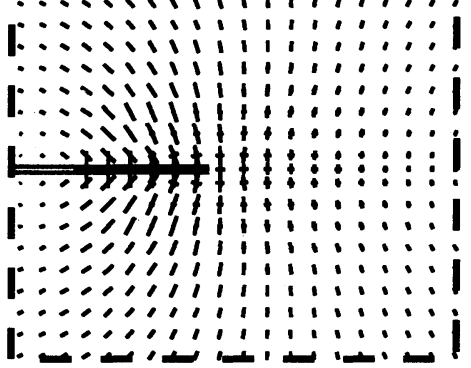

localized crack with bridging

Fig. 7 Distribution of cracking strain and evolution of localized crack (medium size DCB, $a=11.7 \mathrm{~cm}$, $V_{f}=2 \%$ ); (a), (b) contour lines of max. principal cracking strain [\%] at load levels A and B, resp.; (c) principal cracking strain and localized crack in area near original notch tip at load level $B$

3\%. As in the previous analysis, we first determined the material parameters for each volume fraction from its respective uniaxial stress-strain curve; the parameters are listed in Table 1. Then we computed the load-displacement curves of two DCB specimens with different initial notch lengths $a_{1}$ and $a_{2}$ for each volume fraction. To this end we used the same specimen sizes as in the experimental study, i.e., 


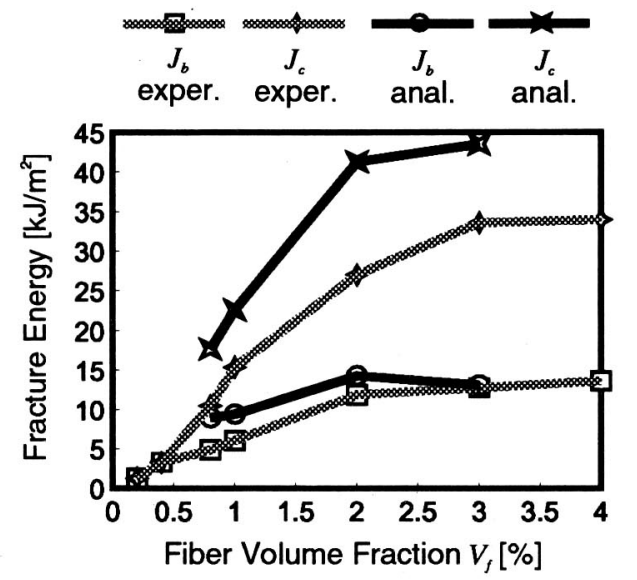

Fig. 8 Effect of fiber volume fraction on the total $\left(J_{c}\right)$ and bridging $\left(J_{b}\right)$ fracture energy

medium size DCB for $V_{f}$ equal $0.8 \%$ and $1 \%$ and large size DCB for $V_{f}$ equal $2 \%$ and $3 \%$. The exact dimensions of each specimen are listed in Table 2. Following the same procedure as in the experimental study ${ }^{8)}$, we calculated the fracture energies. The analytical results are compared with the experimental ones in Fig. 8. The figure shows that the proposed model reproduces the feature that with increasing fiber volume fraction, the fracture energy initially increases but later becomes saturated. The analytical results also confirm the experimentally discovered feature that the multiple cracking contributes more than half of the total fracture energy for fiber volume fractions $2 \%$ and $3 \%$. The model, however, predicts higher magnitudes of the total fracture energy, which can be attributed to the overestimation of the peak load of the DCB specimens.

\section{(3) Effect of the specimen size}

Li and Hashida ${ }^{3)}$ and Maalej et al. ${ }^{8)}$ also observed that changing the specimen size while keeping the fiber volume fraction constant resulted in change in the fracture energy. Namely, the smaller specimens exhibited lower fracture energy. This phenomenon is explained by the fact that the size of a region that must undergo multiple cracking before a steady state cracking is achieved reaches in ECCs up to several hundred square centimeters. When the specimen size is too small, the specimen boundaries limit the development of multiple cracking, which results in lower fracture energy.

In order to examine whether the proposed model can reproduce this feature, we computed the fracture energies for three different DCB specimen sizes: small, medium and large (see Table 2). The fiber volume fraction used in all of these analyses was $2 \%$.

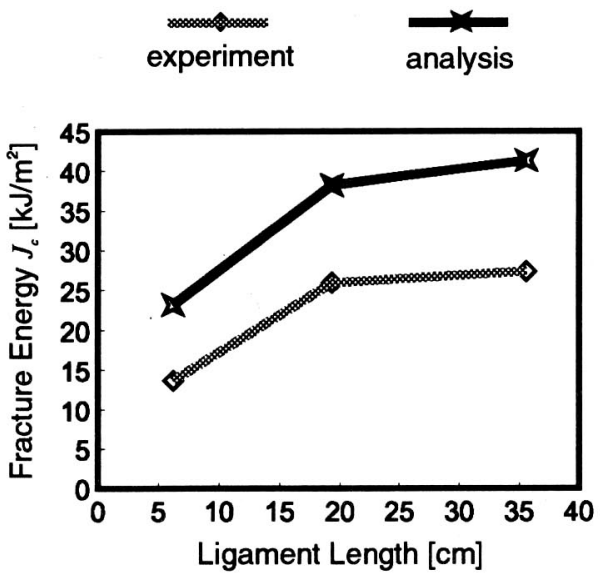

Fig. 9 Effect of specimen size on the total fracture energy $J_{c}$

Fig. 9 compares the analytical and experimental results. Total fracture energy $J_{c}$ is plotted against the initial ligament length $\left(w-a_{1}\right)$, which was selected as a representative of the specimen size. It is seen that the proposed model correctly captures the fact that the small specimen gives value of the fracture energy almost fifty percent lower then the large one. Also consistently with the experimental result the fracture energy for the medium size beam is not much different from that of the large beam.

\section{CONCLUDING REMARKS}

The performance of the proposed analytical model for ECCs has been examined by analyzing fracture behavior of DCB specimens. The present results show that the model fairly well captures the characteristic behavior of ECCs, such as, existence, extent and direction of multiple and localized cracking, trend of the load-displacement curve and effect of fiber volume fraction and specimen size on the fracture energy. The model, however, tends to overestimate the DCB specimens' strength and consequently the fracture energy. The reasons for this tendency may be as follows:

- The number of experimental data used for obtaining the material parameters as well as for the comparison was limited, hence a large error may have been involved. (Only one pair of UT test and DCB test data was available for most of the volume fractions that we examined.)

- The principal stresses in most of the region undergoing the multiple cracking were tensile with minimum principal stress reaching the first crack strength. It is possible that the stress 
transfer capacity of bridging fibers is reduced by the lateral tension. This possibility is currently being studied by analyzing other test specimens, such as, a shear beam.

The agreement between the analytical and experimental results confirmed that the understanding of cracking behavior as outlined in Section 2. of this study is basically correct. However, more experimental investigation is needed to clarify the behavior of ECCs under biaxial loading, namely the effect of lateral stress on the hardening response of multiple cracks and on the composites' strength. If analysis of real structural members is to be carried out, more information about the response of the localized cracks under relative shear displacement is also necessary.

ACKNOWLEDGMENT: The authors wish to express sincere thanks to Professor Victor C. $\mathrm{Li}$ for his helpful discussions and for providing unpublished experimental data.

\section{REFERENCES}

1) Li, V.C.: From Micromechanics to Structural Engineering - The Design of Cementitious Composites for Civil Engineering Applications, Structural Eng./Earthquake Eng. Vol. 10, No. 2, pp.37-48, 1993, Japan Society of Civil Engineers (Proc. of JSCE No. 471/1-24).

2) Li, V.C.: Research Challenges in Toughness Development of Fiber Reinforced Cementitious Composites, Toughening Mechanisms in Quasi-Brittle Materials, S. P. Shah ed., Kluwer Academic Publishers, pp.447-466, 1991.

3) Li, V.C. and Hashida, T.: Ductile Fracture in Cementitious Materials?, Fracture Mechanics of Concrete, Z.P. Bažant ed., Elsevier Applied Science, London and New York, pp.526-535, 1992.

4) Li, V.C: Postcrack Scaling Relations for Fiber Reinforced Cementitious Composites, J. of Materials in Civ. Eng., Vol. 4, No. 1, pp.41-57, 1992.

5) Li, V.C. and Leung, C.K.Y.: Steady State and Multiple Cracking of Short Random Fiber Composites, J. Eng. Mech., Vol. 118, No. 11, pp. 2246-2264, 1992.

6) Hillerborg, A., Modeer, M. and Peterson, P.E.: Analysis of Crack Formation and Crack Growth in Concrete by Means of Fracture Mechanics and Finite Elements, Cement and Concrete Research, Vol. 6, pp. 773-782, 1976.

7) Nanakorn, P. and Horii, H.: A Finite Element with Embedded Displacement Discontinuity, Building for the 21st Century, Y.C. Loo ed., Griffith University, Gold Coast Campus, pp. 33-38, 1995.

8) Maalej, M., Hashida, T. and Li, V.C.: Effect of Fiber Volume Fraction on the Off-Crack-Plane Fracture Energy in Strain Hardening Engineered Cementitious Composites, UMCEE Report No. 94-4, University of Michigan, Ann Arbor, Feb. 1994.

9) Li, V.C., Mishra, D.K., Naaman, A.E., Wight, J.K., LaFave, J.M., Wu, H.C. and Inada, Y.: On the Shear Behavior of Engineered Cementitious Composites, Advn. Cem. Bas. Mat., 1, pp. 142-149, 1994.

10) Chen, W.F. and Han, D.J.: Plasticity for Structural Engineers, Springer-Verlag, New York, 1988.

11) Pamin, J.K.: Gradient-Dependent Plasticity in Numerical Simulation of Localization Phenomena, Delft University Press, Delft, 1994.

12) Horii, H. and Okui Y.: Thermodynamics and Micromechanics-Based Continuum Theory for Localization Phenomena, Fracture of Brittle, Disordered Materials: Concrete, Rock and Ceramics, G. Baker and B.L. Karihaloo eds., E \& FN Spon, London, pp. 391-405, 1995.

(Received June 13, 1995)

\title{
疑似ひずみ硬化を示すセメント系複合材料の破壊挙動に対する解析モデル
}

\author{
Petr KABELE・堀井秀之
}

本論文では短瀻維補強セメント系複合材料の破壊挙動に対する解析モデルの提案を行った.対象とする 複合材料は引張応力下で最初のひびわれ発生後も分散してひびわれが発生し，応力が漸増する疑似ひずみ 硬化挙動を示すものである.複数ひびわれ状態にある材料を均質な連続体とモテル化し,ひびわれの挙動 をクラックひずみにより表し, 弾塑性理論を拡張することにより,その構成式を導入した. 局所化したひ びわれは不連続面として取り扱い,引張軟化関係を満足するものとした.解析モデルを有限要素解析プロ グラムに組み込み, 局所化したひびわれに対しては不連続面を有する有限要素を適用した. 破壊試験の解 析結果は試験結果と良好な一致を示している。 